\title{
Genome-wide identification and expression analysis of calmodulin-like $(C M L)$ genes in Chinese cabbage (Brassica rapa L. ssp. pekinensis)
}

Shanshan Nie, Minjuan Zhang and Lugang Zhang ${ }^{*}$

\begin{abstract}
Background: Calmodulin-like (CML) proteins are a primary family of plant-specific $\mathrm{Ca}^{2+}$ sensors that specifically bind to $\mathrm{Ca}^{2+}$ and deliver a $\mathrm{Ca}^{2+}$ signal. CML proteins have been identified and characterized in many plant species, such as the model plant Arabidopsis and rice. Based on considerable evidence, the roles of CML proteins are crucial in plant growth and development and in the response to various external stimuli. Nevertheless, the characterization and expression profiling of CML genes in Chinese cabbage (Brassica rapa L. ssp. pekinensis) remain limited.

Results: In this study, a genome-wide search and comprehensive analysis were performed, and a total of $79 \mathrm{BrCML}$ genes were identified in Chinese cabbage. Gene structure analysis revealed that these BrCML genes contained two to four conserved EF-hand motifs. Phylogenetic analysis showed that CML homologs between Chinese cabbage and Arabidopsis shared close relationships. The identified BrCML genes were located across ten chromosomes and three different subgenomes of Chinese cabbage. Moreover, 126 pairs of orthologous CML genes were found among Chinese cabbage, Arabidopsis and Brassica oleracea. Expression analysis revealed that the expression of some BrCML genes was tissue-specific and that of some was susceptible to temperature stress. A putative interaction network of BrCML proteins was proposed, which suggested that BrCML2, BrCML6, BrCML15 and BrCML25 were co-expressed and might play roles in flower development and other relevant biological processes of Chinese cabbage.

Conclusions: The results of this study increased the understanding and characterization of $\mathrm{BrCML}$ genes in Chinese cabbage, and will be a rich resource for further studies to investigate $B r C M L$ protein function in various developmental processes of Chinese cabbage.
\end{abstract}

Keywords: CML gene, Chinese cabbage, Expression profiling, Stress response, Interaction network

\section{Background}

Calcium $\left(\mathrm{Ca}^{2+}\right)$, an essential secondary messenger in eukaryotic cells, plays major roles in many aspects of plant growth and development [1-3]. A variety of internal stimuli and external abiotic and biotic stresses, including temperature, light, drought, salinity, plant hormones and disease $[1,4]$, can induce variation in the level of cytoplasmic free $\mathrm{Ca}^{2+}$ and affect the movements of $\mathrm{Ca}^{2+}$ in plant cells. The stress signalling is sensed by

\footnotetext{
*Correspondence: lugangzh@163.com

College of Horticulture, Northwest A\&F University, Yangling 712100, Shaanxi, People's Republic of China
}

unique $\mathrm{Ca}^{2+}$ sensors or $\mathrm{Ca}^{2+}$-binding proteins $[3,5,6]$. $\mathrm{Ca}^{2+}$-binding proteins binding to $\mathrm{Ca}^{2+}$ trigger their conformational changes, and the modulation of activity subsequently regulates downstream targets, thereby transmitting the $\mathrm{Ca}^{2+}$ signals $[3,7,8]$.

$\mathrm{Ca}^{2+}$-binding proteins, which contain the conserved EF-hand motif of a characteristic helix-loop-helix motif, have been identified extensively in plant genomes [7-9]. Calmodulin-like proteins (CMLs) are a large subgroup of plant-specific $\mathrm{Ca}^{2+}$ sensors and are the key components in $\mathrm{Ca}^{2+}$ signal transduction $[7,8]$. CMLs are restricted to plants and differ from Calmodulin (CaM), which is the highly evolutionarily 
conserved $\mathrm{Ca}^{2+}$-binding protein in eukaryotes $[10,11]$. $\mathrm{CaM}$ is composed of four EF-hand domains in plants, whereas CMLs normally possess one to six EF-hands and share $16-75 \%$ amino acid identity with $\mathrm{CaM}[9,11]$. Sequence divergences of EF-hand motifs in CML proteins contribute to the reception of $\mathrm{Ca}^{2+}$ signals from different stimuli and to recognition and activation of the target [3, 8]. Many genes encoding CMLs have been characterized and analysed in the genomes of many plant species. To date, a total of 50 and $32 C M L$ genes have been identified in the model plant Arabidopsis and rice, respectively [12-14]. Additionally, lists of $C M L$ genes were discovered in some vegetable crops, such as tomato, cucumber and common bean $[15,16]$.

Previous studies suggest that different $C M L$ genes likely have distinct and significant physiological roles in a series of developmental processes $[8,9]$. CML24 gene has functions in ion-homeostasis, photoperiod-response and hormone-induced morphogenesis and growth [17]. Arabidopsis CML23 and CML24 play roles in the transition to flowering of Arabidopsis [17, 18]. Notably, many $C M L$ genes are crucial for the growth of pollen and pollen tubes [19-22]. For example, the loss-offunction of CML24 and CML25 in Arabidopsis mutants strongly affected pollen germination and pollen tube growth [22, 23]. Moreover, CMLs acting as key players in $\mathrm{Ca}^{2+}$ signalling are also involved in the complex signalling pathways responding to abiotic and biotic stresses [8]. In Arabidopsis, AtCML39 is implicated in the transduction of light signals and the promoting of seedling establishment [24]. The knockout mutants of AtCML9 displayed functions that increased plant tolerance to drought and salinity stress [25]. The CML24 gene also functions in the inhibition of pathogen-induced NO generation [26]. Furthermore, many reports have suggested the roles of CMLs in hormone homeostasis and signalling [3, 8].

Chinese cabbage (Brassica rapa L. ssp. pekinensis), a member of the Brassicaceae family, is an important leaf vegetable crop that is grown worldwide. A comparative genomic analysis revealed the close relationship between Chinese cabbage and Arabidopsis. The whole genome data of Chinese cabbage provide useful resource for the analysis of $\mathrm{Ca}^{2+}$-binding proteins including, CaM, CML and calcium-dependent protein kinase (CDPK) in Chinese cabbage [16, 27]. Although $36 C M L$ genes have been characterized in B. rapa using the BLASTP program [16], some potential candidate $C M L$ genes and their roles require further exploration. To date, systematic expression analyses of CMLs have not been conducted in Chinese cabbage. Therefore, the comprehensive identification and expression analysis of $C M L$ genes in Chinese cabbage could uncover the molecular mechanisms responsible for responses to abiotic and biotic stresses. In this study, to further systematically explore the $C M L$ genes in Chinese cabbage, genomic analysis and similarity searching of the conserved EF-hand domain against the whole genome of Chinese cabbage were performed. The primary aims of this study were to identify the potential $B r C M L$ genes at the whole-genome level and then analyse their gene structure, chromosomal distribution and orthologous genes. Moreover, the expression profiles of $B r C M L$ genes were investigated in different tissues and under various stress treatments. A putative interaction network of BrCML proteins was proposed to explore their roles in plant development and stress responses in Chinese cabbage. The outcomes of this study provide insights into the understanding of $B r C M L$ genes in Chinese cabbage and should facilitate the discovery of more $C M L$ genes in other Brassicaceae crops.

\section{Results}

\section{Identification and characterization of $B r C M L$ genes in} Chinese cabbage

To identify the putative $C M L$ genes of Chinese cabbage, 50 CML protein sequences of Arabidopsis [12] were retrieved and used as the query to search against BRAD and NCBI databases using the BLASTP program. As a result, 79 protein sequences in Chinese cabbage were obtained and subjected to Pfam, SMART analysis and InterProScan sequence searching. These identified genes were named, in order, from BrCML1 to $B r C M L 50$ (Additional file 1: Table S1). Among these $B r C M L$ genes, BrCML8-2/BrCML8-3, BrCML11-2/BrCML11-3, BrCML22-1/BrCML22-2 and BrCML41-1/BrCML41-2 were different transcript variants or isoforms from the same gene. The length of protein sequences ranged from 100 (BrCML9-2) to 411 (BrCML50-2) amino acids. Furthermore, the physical and chemical characteristics of $79 \mathrm{BrCMLs}$ were analysed (Additional file 1: Table S1). The molecular weights of the proteins ranged from 11.078 to $43.862 \mathrm{kDa}$, and the theoretical pI values ranged from 4.02 to 9.02 . More detailed information, including the instability index, aliphatic index and grand average of hydropathicity (GRAVY), is provided. Additionally, compared with the previous report, we found that $22 \mathrm{BrCML}$ genes were same as the results by Mohanta et al. [16], and the repeated gene were marked and listed in Additional file 1: Table S1.

To obtain the characterization and subfamily classification of the $79 \mathrm{BrCML}$ proteins, their amino acid sequences were used to generate a phylogenetic tree by the NJ Bootstrap method (Fig. 1a). All BrCMLs were classified into six subfamilies (groups I-VI). The sequence similarities of members in each subgroup were relatively high. Moreover, the exon-intron structures were analysed to examine the structural diversity of $B r C M L$ genes (Fig. 1b). Most of the genes had 


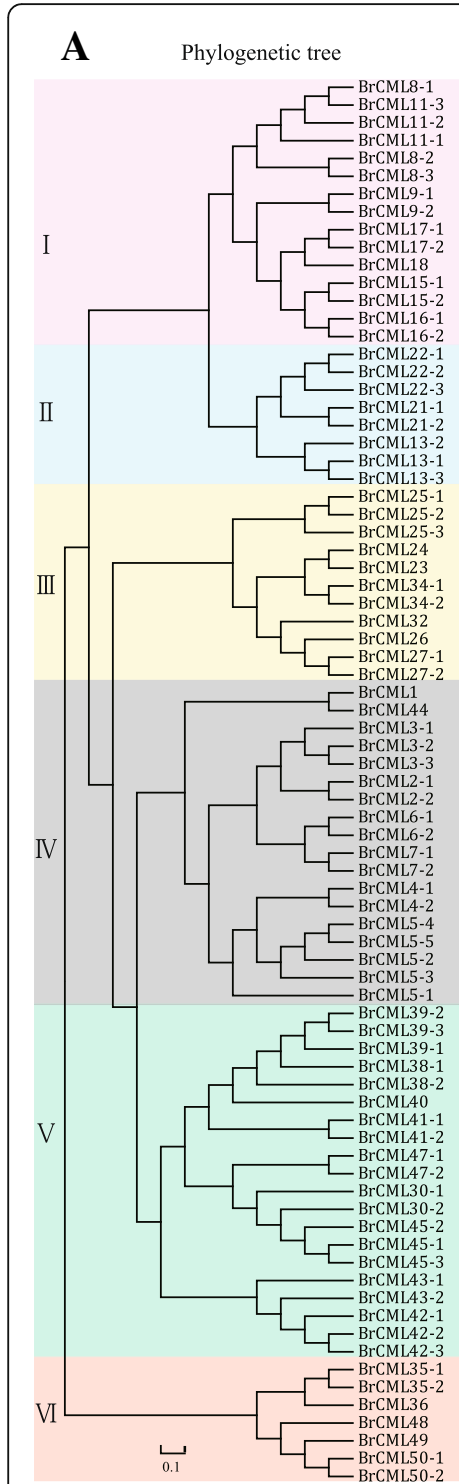

B

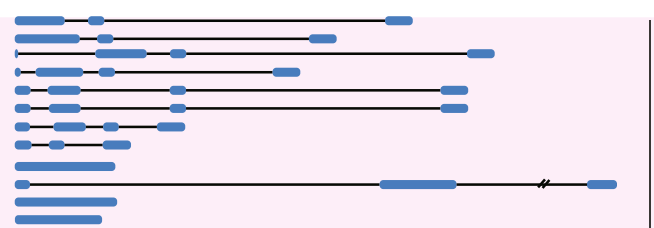

$\longrightarrow$

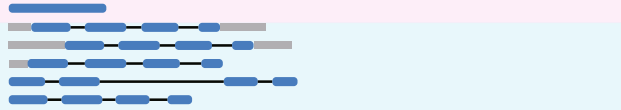

$\longrightarrow$

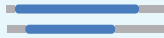

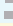
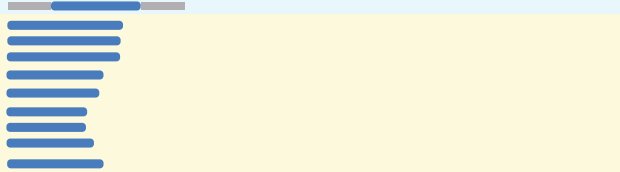

2

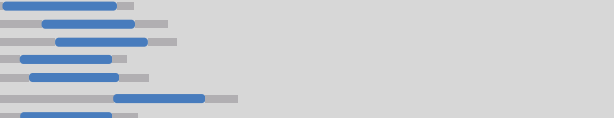

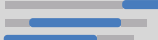

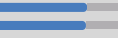

2

$\longrightarrow$

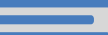

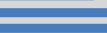

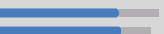

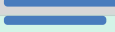

프

는

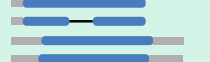

$-$

2

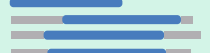

2

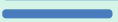

$\longrightarrow$

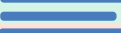

2

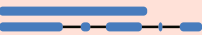

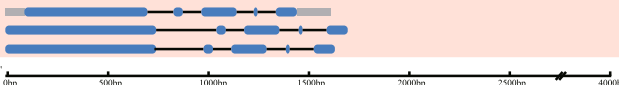

s"ibp $\quad$ CDS UTR - Intron
C EF-hand motif composition

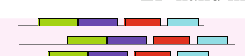

$\sqrt{1}$

$\longdiv { 1 \quad r + 2 }$

$\longdiv { \square }$

$\longdiv { + \square }$

$\square \square$

$\sqrt{\square+r}$

$+1$

$+\square$

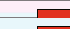

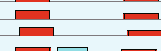

ए

$\longrightarrow$

$\sqrt{\square} \sqrt{\square}$

$\square+\square$
$\square+\square$

$\longdiv { \square }$

$\sqrt{\square+2}$

$\square+\square$

$\sqrt{15 \sqrt{2}}$

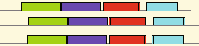

$\sqrt{\square \quad \square}$

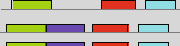

$\square$

$\lcm{\square}$

$\square+\square$

$+\square$

$\square$

$\longdiv { \square }$

मே㇒

$+r$

$\longdiv { 1 } \square$

$\longdiv { \square }$

$\square \square$

म

$\square \square$

$\square \square$

$\longdiv { \square \square }$

$\sqrt{\square}$

मा

मr

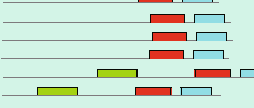

म

म

r

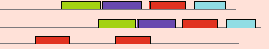

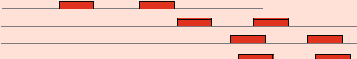

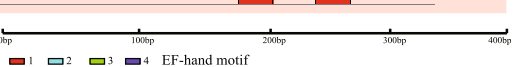

Fig. 1 Characterization of the identified BrCMLs in Chinese cabbage. a Phylogenetic relationships and classification of BrCML proteins. b The exon-intron structure distribution of BrCML genes. c Distribution of conserved EF-hand motifs among the BrCML proteins

a single exon without intron region, particularly some of the genes in groups III, IV and V. To further analyse the features of the $79 \mathrm{BrCML}$ proteins, the MEME tool was used to search and predict their conserved domains. As the results display, the BrCML proteins contained two to four highly conserved EF-hand motifs (Fig. 1c). The LOGO of four amino acid motifs was also generated (Additional file 2: Figure S1). In general, the genes in the same subgroup shared a close phylogenetic relationship, high sequence similarity and similar gene structures.

\section{Phylogenetic relationships of CMLs among Chinese cabbage, Arabidopsis and rice}

The protein sequences of the 79 BrCMLs in Chinese cabbage and 50 AtCMLs in Arabidopsis were obtained in this study. The evolutionary relationships and classification of CMLs between Chinese cabbage and Arabidopsis were analysed based on the full-length amino acid sequences (Fig. 2). The constructed phylogenetic tree showed that the CML proteins were classified into seven subgroups (groups I-VII). Most CMLs were in group VI, followed by groups IV andI, with the relatively few genes in groups II, III and VII. Notably, 


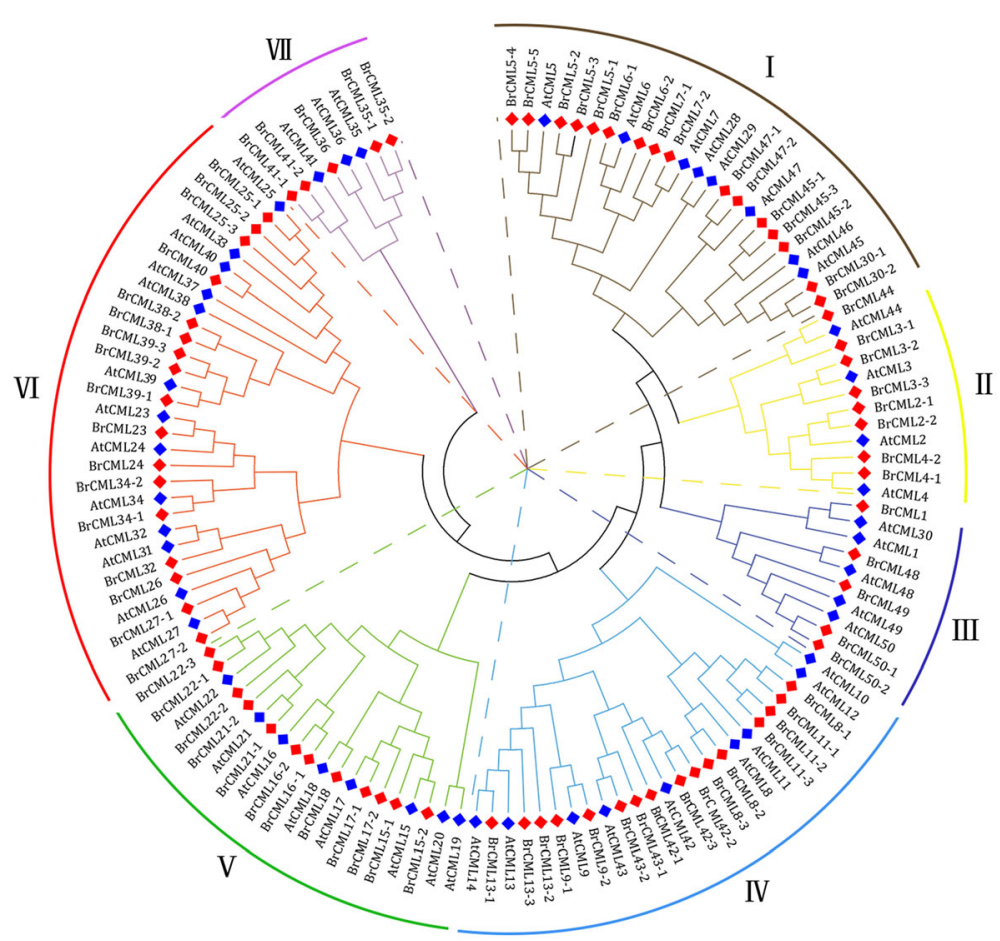

Fig. 2 Phylogenetic tree of CML proteins in Chinese cabbage and Arabidopsis constructed with the neighbourhood-joining method

group VII was the smallest group and contained only five BrCMLs and three AtCMLs. Moreover, the amino acid sequences of 32 OsCMLs on rice were downloaded from the rice genome database (TIGR), and a more detailed phylogenetic analysis of CMLs was performed among Chinese cabbage, Arabidopsis and rice (Additional file 3: Figure S2). The evolutionary relationships suggested that most $B r C M L$ genes were closely related to their corresponding homologous genes in Arabidopsis and rice.

\section{Chromosomal localization and orthologous gene analysis of $B r C M L$ genes}

To examine the chromosomal distribution of the 79 $B r C M L s$, the genes were mapped onto the chromosomes of Chinese cabbage against the $B$. rapa genome database (chromosome v1.5). In total, $75 \mathrm{BrCML}$ genes were separately distributed on the ten chromosomes (Fig. 3), whereas four genes (BrCML3-1, BrCML6-2, BrCML133 and $B r C M L 17-2$ ) could not be assigned to any specific chromosome. Most $B r C M L$ genes were located on chromosome Br03, whereas a single BrCML50-1 was found on chromosome Br10. Furthermore, the $B r C M L$ genes were anchored on the three fractionated subgenomes of the B. rapa genome, including the least fractionated (LF) subgenome, the medium fractionated (MF1) subgenome and the most fractionated (MF2) subgenome [28], with $27 \mathrm{BrCMLs}$ fractionated into the LF subgenome, 23 into the MF1 subgenome, and 25 into the MF2 subgenome (Fig. 3; Additional file 4: Table S2). Moreover, 21 pairs of $B r C M L$ syntenic paralogs were found on different subgenomes of Chinese cabbage (Additional file 4: Table S2). For example, BrCML15-1/ Bra025896 and BrCML15-2/Bra031033 were located in the LF and MF2, respectively, and both exhibited high sequence similarities with AtCML15 (AT1G18530). The syntenic analysis of these $B r C M L$ paralogs was performed, and the results were shown in Fig. 4.

Furthermore, in this study, comparative analysis was used to identify orthologous $C M L$ genes among Chinese cabbage, $A$. thaliana and $B$. oleracea. As a result, 64 and 62 pairs of orthologous $C M L s$ were identified between Chinese cabbage and A. thaliana and between Chinese cabbage and $B$. oleracea, respectively (Fig. 4; Additional file 5: Table S3). Among these orthologous $C M L s$, most $B r C M L$ genes were found with 1-6 orthologous genes in A. thaliana and B. oleracea. Three genes (BrCML9-1, BrCML24 and BrCML32) had no orthologue in Arabidopsis, and two genes (BrCML8-2 and BrCML17-1) had no orthologue in B. oleracea. Additionally, several orthologous genes of $B r C M L 8-1$ and $B r C M L 8-2$ belonged to Arabidopsis CaM genes (Additional file 5: Table S3), suggesting the close genetic relationship and most likely similar function between $C M L$ and $C a M$ genes. The orthologous genes should provide a strong resource and reference for 


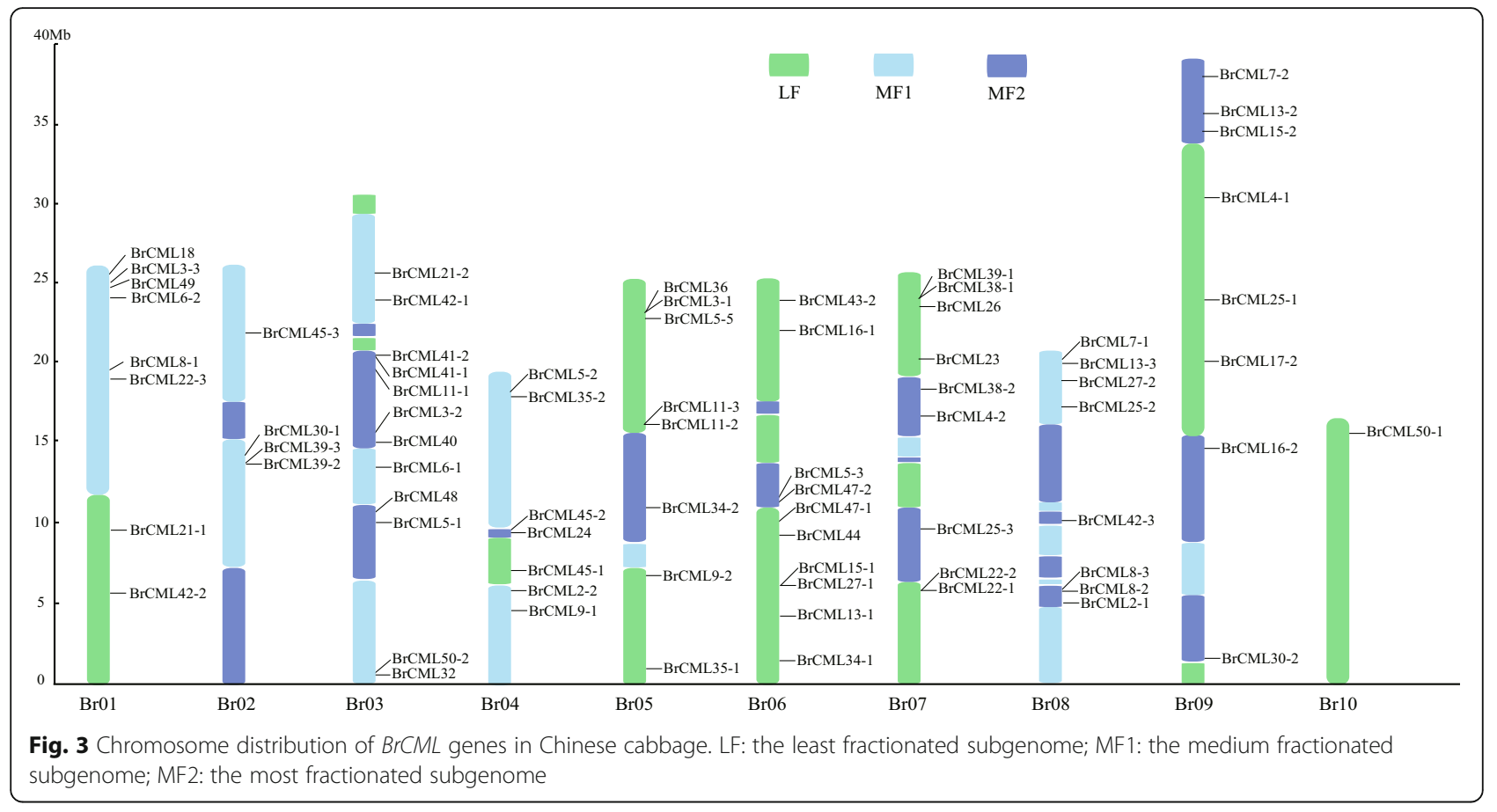

exploring the functions and roles of BrCMLs in Chinese cabbage.

\section{Expression profiling of $\mathrm{BrCML}$ genes in different tissues and under stress treatments}

To detect the tissue-specific expression profiling of the $B r C M L$ genes, the gene expression FPKM values of 71 sequences in five different tissues were calculated by exploiting the previously reported RNA-Seq data on Chinese cabbage 'Chiifu' [29]. The map of the expression profiling for the $B r C M L s$ was prepared using the $\log _{2}$ FPKM values (Fig. 5a). The $\operatorname{BrCML}$ genes exhibited differential expression in root, stem, leaf, flower and silique of Chinese cabbage, although the expression of
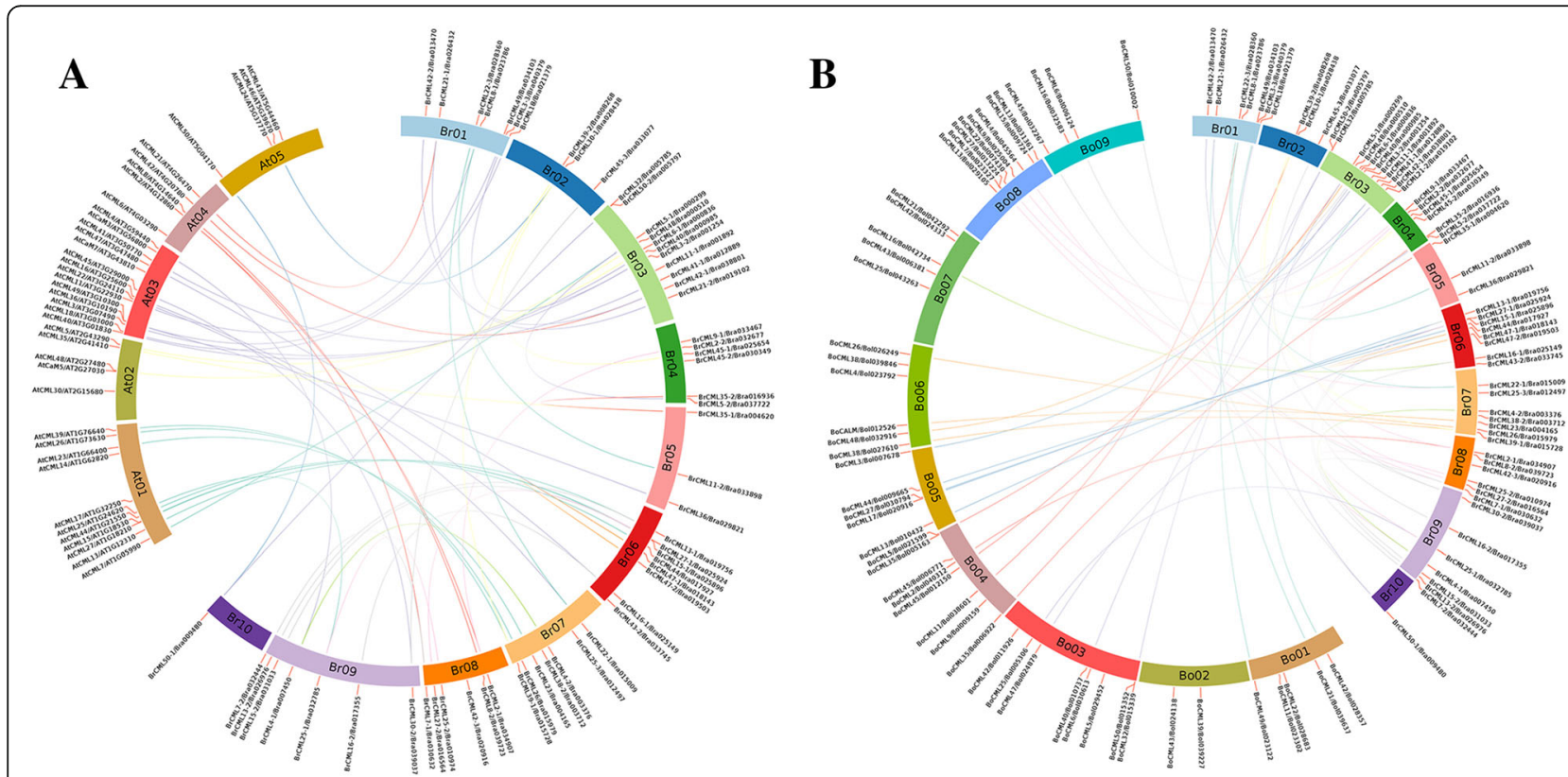

Fig. 4 Syntenic analysis of CML genes in Chinese cabbage, Arabidopsis thaliana and Brassica oleracea. a The orthologous and paralogous BrCML genes were mapped onto the chromosomes of Chinese cabbage (Br01-Br10) and Arabidopsis (At01-At05). $\mathbf{b}$ The orthologous and paralogous BrCML genes were mapped onto the chromosomes of Chinese cabbage (Br01-Br10) and Brassica oleracea (Bo01-Bo09) 
A
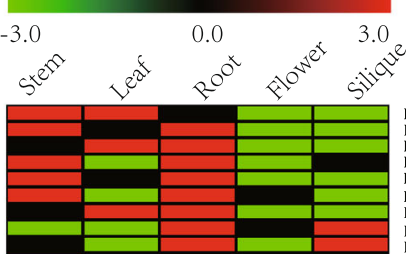

BrCML24
BrCML38-1 BrCML38-1
BrCML27-2
BrCML23 BrCML23
BrCML27-1 BrCML26 BrCML4-1

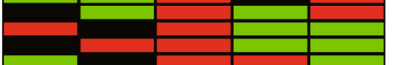

BrCML43-1
BrCML38-2

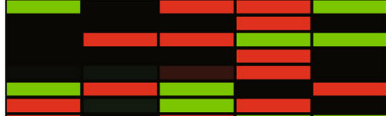
BrCML1
BrCML6-1
BrCML42-1 BrCML42-1
BrCML7-1 BrCML25-1 BrCML35-1 BrCML35-2 BrCML13-1 BrCML30BrCML9-1
BrCML22-1
BrCML42-2 BrCML49 BrCLL8-
BrCML16-2 BrCML16-2 BrCML11-2 BrCML18 BrCML13-2
BrCML43-2 BrCML39-3
BrCML8-2 BrCML8-2
BrCML11-1 BrCML21-2 BrCML39-2 BrCML47-1 BrCML34-2
BrCML21-1 BrCML44
BrCML25-3 BrCML25-3
BrCML42-3 BrCML30-1 BrCML32 BrCML34-1
BrCML22-3 BrCML22-3
BrCML15-1 BrCML45-2 BrCML3-2
BrCML48 BrCML48
BrCML2-1 BrCML2-1
BrCML2-2
BrCML3-3 BrCML3-3 BrCML15-2 BrCML25-2
BrCML47-2 BrCML50-1 BrCML5-1 BrCML4-2 BrCML36 BrCML50-2

\section{C}
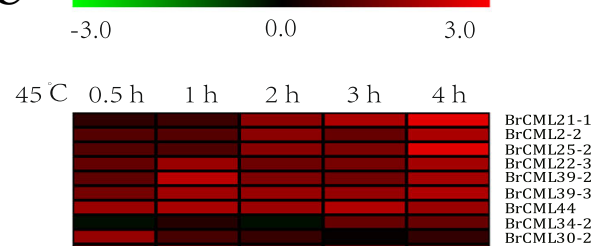

rCML25-2
rCML22-3
rCML39-2

BrCML39-2
BrCML39-3
BrCML44

$\mathrm{BrCML} 44$
$\mathrm{BrCML34-2}$

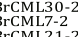

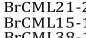



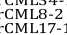

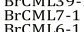

CML15-2

BrCML9-1

BCML9

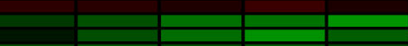

$\mathrm{BrCML26}$
$\mathrm{BCCML} 26$
$\mathrm{BC} C \mathrm{CML}$

BrCMLA35-1

BrCMLA1-1
BrCML4-2

BrCML44-2
BrCML2-2
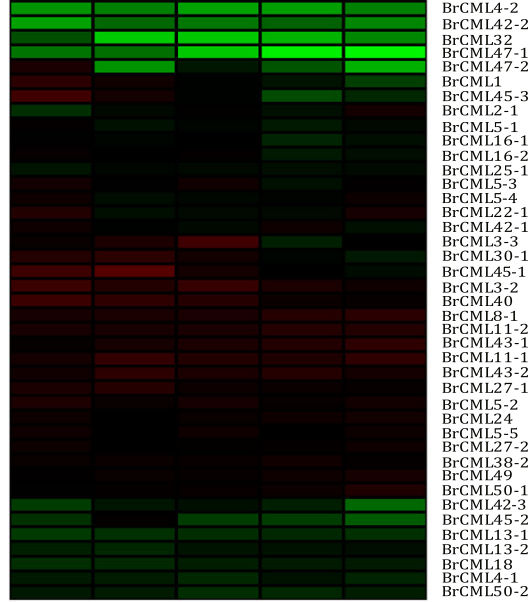

BrCML45-3
BrCML2-1

BrCML5-1

BrCML16-1
BrCCL16-2
BrC

BrCML25-1

BrCML5-4
BrCML22-1

BrCML42-1

BrCML45-1

BrCML3-2
BrCML40

.

BrCMLA1-
BrCML11-

BrCML43-2

$\mathrm{BrCML5}-2$
BrCML24

BrCML5-5
BrCML27-2

BrCML38-2

BrCML50-1
BrCML42-3

BrCML45-2

PrCML13-1
BrCML13-2

rCML18
rCML4-1

B

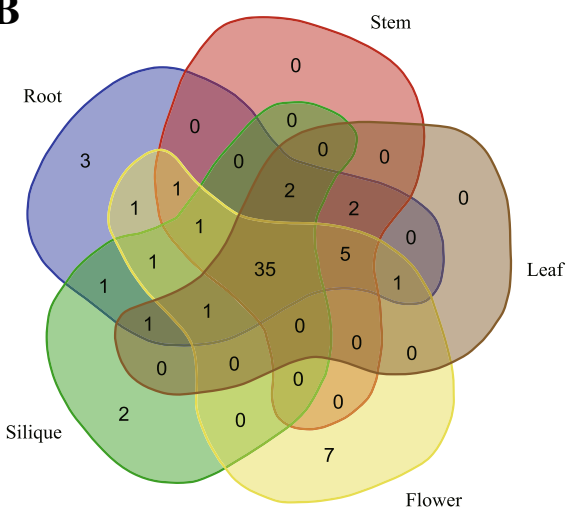

Fig. 5 Expression analysis of BrCML genes in Chinese cabbage. a Differential expression patterns of BrCML genes in root, stem, leaf, flower and silique of Chinese cabbage. $\mathbf{b}$ Venn diagram of BrCML expressions in five tissues of Chinese cabbage. $\mathbf{c}$ Differential expression patterns of BrCML genes under $45^{\circ} \mathrm{C}$ heat stress for $0.5,1,2,3$ and $4 \mathrm{~h}$

eight genes was not found in any tissue. $\operatorname{BrCML24}$ had the highest levels of expression in stem, leaf, flower and silique and was second only to $B r C M L 38-1$ in the root (Additional file 6: Table S4). Venn diagram of the expression analysis revealed that 35 of the $B r C M L s$ were expressed in five tissues, whereas several genes were specifically expressed in a single tissue (Fig. 5b). For example, BrCML8-2, BrCML39-2 and BrCML39-3 were only expressed in the root, and $B r C M L 32$ and $B r C M L 34-1$ were only expressed in the silique. Remarkably, seven genes (BrCML2-2, BrCML3-3, BrCML6-1, BrCML7-1, BrCML15-1, BrCML15-2 and BrCML25-3) were only expressed in flower and considered as flowerspecific expressions, implying that they play vital roles in 
the development of floral organs or other relevant biological processes.

The relative expression levels of all the identified $B r C M L$ genes were also validated under temperature stress. In this study, the expression values of $B r C M L$ genes under $45^{\circ} \mathrm{C}$ heat stress were obtained from a microarray study with the Chinese cabbage 'Chiifu' [30]. A total of 71 $B r C M L$ genes were analysed and their fold changes under different treatments were counted (Additional file 7: Table S5). These genes showed diverse dynamic expression patterns in response to $45^{\circ} \mathrm{C}$ heat stress with exposure at 0.5 , 1, 2, 3 or $4 \mathrm{~h}$ (Fig. 5c). In the five treatment groups under $45^{\circ} \mathrm{C}$ heat stress, the expression of 38 and $18 \mathrm{BrCML}$ genes was up-regulated and down-regulated, respectively. Differential expression levels of most genes were less than 2-fold under treatment (Additional file 7: Table S5). Furthermore, the differential expression of $B r C M L$ genes related to the formation of leafy-head in response to low temperature was analysed. The transcriptomic data of Chinese cabbage inbred line 'Chiifu-402' were retrieved from a previous report concerning low-temperature induced leafy-head formation [31]. Consequently, 54 differentially expressed $B r C M L$ genes were detected between the heading group (a constant $25^{\circ} \mathrm{C}$ temperature) and non-heading group (a $4{ }^{\circ} \mathrm{C}$ low temperature treatment) (Additional file 8: Figure S3; Additional file 9: Table S6).

\section{Identification of miRNAs targeting BrCML genes}

MiRNAs are known to regulate the expression of corresponding target genes [32]. To identify the putative miRNAs targeting $B r C M L$ genes, the data of several miRNA libraries were obtained from previous reports on miRNA identification in Chinese cabbage. The psRNATarget program performed the target prediction of miRNAs. In this study, six known miRNAs and one novel miRNAs (named miRN01) were predicted to potentially target 10 $B r C M L$ genes (Additional file 10: Table S7). The putative regulatory relationships of 10 miRNA- $B r C M L$ pairs (Additional file 11: Figure S4) were described using Cytoscape software. Moreover, miR835 and miR838 separately targeted two different $B r C M L$ genes, and miRN01 targeted two syntenic genes of BrCML25.

\section{The interaction networks of BrCML proteins in Chinese cabbage}

In this study, 79 BrCML proteins were screened in Chinese cabbage, most of which shared close relationships with their homologs or orthologues in Arabidopsis. To validate the protein interactions of BrCMLs, STRING software was used to construct a putative interaction network according to the orthologous proteins in Arabidopsis (Fig. 6). A total of 63 BrCML proteins associated with 28 known Arabidopsis proteins were involved in the network, whereas 15 BrCMLs and 9 proteins of
Arabidopsis were not found in associations with any other proteins (Fig. 6; Additional file 12: Table S8). A central node (AT3G10300) was associated with the most proteins, including $38 \mathrm{BrCML}$ and 15 Arabidopsis CML proteins. The protein-protein associations revealed that some BrCML proteins were most likely co-expressed according to Arabidopsis CML proteins. Of note, BrCML2, BrCML6, BrCML15 and BrCML25 formed a close protein interaction and showed putative co-expression and cooccurrence (Fig. 6). The analysis of interaction network suggested that several BrCML proteins could interact with one another and regulate downstream proteins, thereby, enhancing the understanding of BrCML protein functions in Chinese cabbage.

\section{Discussion}

Plant $\mathrm{Ca}^{2+}$ sensor proteins involved in $\mathrm{Ca}^{2+}$ signalling networks are crucial proteins governing $\mathrm{Ca}^{2+}$ homeostasis during multiple cellular processes $[1,2]$. The CMLs are a primary group of unique EF-hand proteins in plants that bind to $\mathrm{Ca}^{2+}$ and regulate downstream targets in response to various stimuli-induced $\mathrm{Ca}^{2+}$ fluctuations and signalling transduction $[3,6,8]$. A series of CML proteins and an increasing number of target genes have been identified in many plant species. For example, 50 and 32 CML genes have been found in Arabidopsis and rice, respectively [12-14]. Chinese cabbage is one of the important leaf vegetables with high nutritional and economic value and is widely grown around the world. Nevertheless, the characterization and expression analysis of $B r C M L$ genes in Chinese cabbage have not been studied. In this study, homology searching against the BRAD and the NCBI database was performed and 79 $B r C M L$ genes were identified in Chinese cabbage, a number comparable with that in previous reports in the model crop Arabidopsis [12, 13, 16]. To obtain a comprehensive overview and characterization of these $B r C M L$ genes, the biochemical properties, structural features and expression patterns were explored to provide a rich source for further functional studies of $B r C M L s$ in Chinese cabbage.

As demonstrated by the evolutionary conservation in multiple species, $\mathrm{CaM}$ proteins have important functions in response to different developmental and environmental signals $[10,11,13]$. Plant CMLs have at least $16 \%$ sequence identity with the conserved $\mathrm{CaM}$ proteins and display a diversity of protein structures and expression features $[8,9,12]$. The structural variation of CML proteins may confer functional diversity, and the number and sequence variation of EF-hands among CML members are implicated in the $\mathrm{Ca}^{2+}$ binding properties and differential responses $[3,10,13]$. In this study, two to four conserved EF-hand motifs were typically found in the identified $B r C M L$ genes (Fig. 1c), which is consistent 


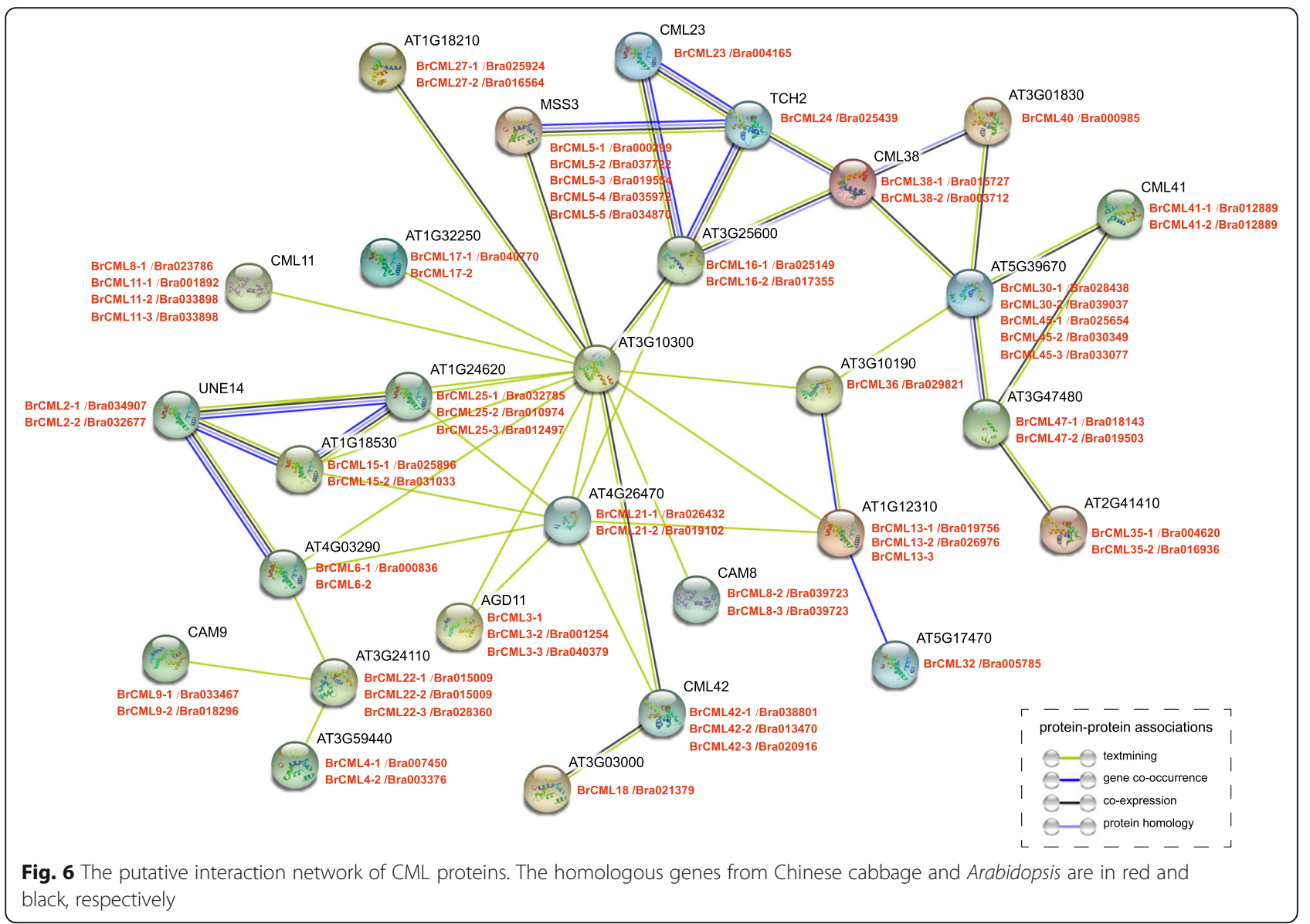

with the typical character of CML proteins [10]. The intron-exon structure analysis showed that most $C M L$ genes were intronless, which is in agreement with the previous reports $[9,12,16]$. Some $B r C M L$ genes that shared close phylogenetic relationships displayed similar EF-hand distributions and intron-exon structures, which indicate that these genes may have similar biological roles and expression characteristics. Phylogenetic analysis of $C M L$ genes between Chinese cabbage and Arabidopsis showed that many $B r C M L$ genes had high similarities with their corresponding Arabidopsis homologs. Moreover, most of the identified $B r C M L$ genes were found as orthologous genes in Arabidopsis and $B$. oleracea, suggesting the conservation of $C M L$ genes among Brassicaceae crops. The current evidence indicates that Brassica species underwent an ancestral whole-genome triplication from their diploid progenitors $[33,34]$. Comparative analysis of Brassica genomes demonstrates that the $B$. rapa genome evolved from a hexaploid progenitor with a triplicated diploid ancestral genome $[28,34]$. In the present study, we assigned the $79 \mathrm{BrCML}$ genes across the three different subgenomes of Chinese cabbage (Fig. 3), which showed a relatively uniform distribution with 27 genes in the LF, 23 in the
MF1 and 25 in the MF2. We also identified 21 pairs of $B r C M L$ syntenic paralogs, supporting the extensive genome triplication of Chinese cabbage [28].

The spatio-temporal expression pattern of CMLs is considered a critical index for their function annotations [3, 8]. Many reports demonstrate that $C M L$ genes are differentially expressed in different plant tissues, developmental stages and cell type and in response to various external stimuli $[13,35]$. Expression profiling of $C M L$ genes is extensively validated in many species, including pea, tomato, strawberry, Arabidopsis and rice $[15,19,35-37]$. As expected, our results revealed that the expression of $B r C M L$ genes differed distinctly among five Chinese cabbage tissues, with several $B r C M L$ genes exhibiting tissue-specific expression patterns (Fig. 5). Notably, the expression of $B r C M L 2-2$, BrCML6-1, BrCML15-1, BrCML15-2 and BrCML25-3 was specific to the flower of Chinese cabbage. Moreover, the interaction network of $B r C M L$ genes suggested that BrCML2, BrCML6, BrCML15 and BrCML25 were co-expressed based on reference to the homologs in Arabidopsis, implying that these BrCMLs may participate in the same regulatory pathways and are capable of similar functions during plant 
development and stress responses. Transcriptomic analysis of Arabidopsis showed that a set of CMLs (e.g., CML2, CML6, CML15, CML25, CML42 and CML49) were significantly up-regulated during pollen germination or pollen tube growth [17]. Furthermore, AtCML25 mediates the regulation of $\mathrm{Ca}^{2+}$ and $\mathrm{K}^{+}$ transmembrane trafficking in pollen grains and pollen tubes and acts as an important transducer during pollen germination and tube elongation [21]. This evidence could support a hypothesis that BrCML2, BrCML6, BrCML15 and BrCML25 may exert functions in pollen germination and pollen tube growth of Chinese cabbage. Additionally, in this study, the candidate miRN01 possibly negatively targeted $B r C M L 25-1 /$ Bra032785 and BrCML25-2/Bra010974 (Additional file 10: Table S7). Functional analysis of miRNAs could greatly increase the understanding of $C M L$ gene functions.

The expression of $C a M$ and $C M L$ genes, and alteration of intracellular $\mathrm{Ca}^{2+}$ gradients, varies specifically in response to $\mathrm{Ca}^{2+}$ signalling and a variety of stress responses, including biotic and abiotic stresses [7, 8, 13]. In Arabidopsis, AtCML37, AtCML38 and AtCML39 respond to several developmental and stimulus-induced signalling pathways [23, 38], and AtCML42 and AtCML43 may function to increase resistance to pathogens $[39,40]$. Additionally, CaM1 in pepper and CaM13 in tobacco play vital roles in virus-induced plant immune responses [41, 42]. The mutant of AtCaM3 showed reduced thermotolerance and the downregulation of heat-related proteins [43, 44]. According to the summary of Delk et al. [17], the expression levels of CML24 are related to responses to hormones, cold, and heat, among others. In this study, $45^{\circ} \mathrm{C}$ heat stress induced an increase in transcript levels of many $B r C M L$ genes, particularly for $B r C M L 21-1$, which exhibited the highest fold change in expressions. Moreover, $\mathrm{BrCML} 44$ showed reverse patterns of expression under $45^{\circ} \mathrm{C}$ and $4{ }^{\circ} \mathrm{C}$ treatments, indicating that the expression was susceptible to heat stress or low temperature. These results will contribute to further functional explorations of $B r C M L$ genes in Chinese cabbage.

\section{Conclusions}

In this study, a total of $79 \mathrm{BrCML}$ genes were identified in Chinese cabbage at the whole-genome level. Comprehensive analysis and expression profiling of $B r C M L$ genes were performed to determine the potential functions in $\mathrm{Ca}^{2+}$ signalling networks and in response to various stresses and environmental stimuli. The $\operatorname{BrCML}$ genes contained two to four conserved EF-hand motifs and shared close relationships with their homologs in Arabidopsis. We also found a series of orthologous $B r C M L$ genes in Arabidopsis and B. oleracea. Furthermore, expression analysis revealed the tissue-specific expression and temperature susceptibility of $B r C M L$ genes. Several genes, including $B r C M L 2-2, B r C M L 6-1, B r C M L 15-1$, $B r C M L 15-2$ and $B r C M L 25-3$, showed flower-specific expression patterns. Remarkably, a protein interaction analysis showed that BrCML2, BrCML6, BrCML15 and BrCML25 were likely co-expressed, suggesting that these proteins have critical functions in biological processes relevant to Chinese cabbage flower development. These results will provide a rich resource for further functional studies of BrCML proteins in Chinese cabbage.

\section{Methods}

\section{Identification of $B r C M L$ genes in Chinese cabbage}

To identify the $C M L$ genes in Chinese cabbage, the reported CML gene sequences in Arabidopsis thaliana were downloaded from the TAIR database (The Arabidopsis Information Resource) and used as a query to perform BLASTP searching. The whole genome sequences of Chinese cabbage from the BRAD database (Brassica database) [45] and the NCBI database were used to search the potential $C M L$ genes in Chinese cabbage. To differentiate between $C a M$ and $C M L$ genes which were homologs in Chinese cabbage, we follow the principle of the major character of EF-hand containing proteins $[9,11,15,16,37]$ and screen the candidate $C M L$ genes. The candidate proteins of $C M L$ genes were further confirmed by analysing the EF-hand motifs through the use of public databases including NCBI Conserved Domain Database (http:// www.ncbi.nlm.nih.gov/cdd), Pfam (http://pfam.xfam.org/), InterProScan (http://www.ebi.ac.uk/Tools/pfa/iprscan5/) and SMART (http://smart.embl-heidelberg.de/), and the domain ID of searching EF-hand was cd00051, PF00036, IPR002048 and SM00054 in each database, respectively. The identified candidate genes were named from $B r C M L 1$ to $B r C M L 50$ according to homologous genes and annotation descriptions. Their gene sequences and deduced amino acid sequences were retrieved for the following characterization of $\mathrm{BrCML}$ genes.

\section{Analysis of conserved domain, gene structure and characterization of $\mathrm{BrCML}$ genes}

The BrCML protein sequences were analysed for physical and chemical characteristics, including the molecular weight (MW), theoretical point (pI), instability index, aliphatic index and grand average of hydropathicity (GRAVY), using the ProtParam tool of ExPASy (http:// web.expasy.org/protparam/). The exon-intron structure analysis of $B r C M L$ genes was conducted using the GSDS 2.0 (Gene structure display server; http://gsds.cbi.pku.edu.cn//index.php) program with default parameters. The conserved motifs were analysed using the MEME tool (version 4.11.4; http://meme-suite.org/tools/meme), with the minimum width of motifs as 10 , the maximum width 
of motifs as 40 and the other parameters as default values. The LOGO of conserved motifs was described according to the motif analysis results.

\section{Phylogenetic relationships of CML proteins in Chinese cabbage, Arabidopsis and rice}

The multiple alignments of BrCML proteins were performed using ClustalX with default parameters. The reported 32 OsCMLs in Oryza sativa L. [14] were downloaded from the rice genome database (The Institute for Genomic Research, TIGR; http://rice.plantbiology.msu.edu/). Phylogenetic analysis of CML proteins in Chinese cabbage, Arabidopsis and rice was performed using MEGA 7.0 [46] with the neighbourhood-joining (NJ) method and bootstrap values of 1000 replicates.

\section{Identification of orthologous $B r C M L$ genes and syntenic analysis in Chinese cabbage}

According to the BRAD and chromosome data (v1.5) of Chinese cabbage, the identified $B r C M L$ genes were located into ten chromosomes and three fractionated subgenomes. Chromosomal positions of $B r C M L$ genes were analysed and presented using MapInspect Software. The syntenic genes of $B r C M L s$ were identified in online searching on the BRAD (http://brassicadb.org/brad/searchSynteny.php). The orthologous $B r C M L$ genes among Chinese cabbage, A. thaliana and Brassica oleracea were also searched. Cricos software [47] was employed for syntenic analysis of orthologous and paralogous CML genes among Chinese cabbage, $A$. thaliana and $B$. oleracea.

\section{Expression pattern analysis of $B r C M L$ genes and interaction networks}

To analyse the expression patterns of $B r C M L$ genes in Chinese cabbage, the Illumina RNA-Seq data of $B$. rapa 'Chiifu' [29] were downloaded and used for gene expression profiling in five tissues that included root, stem, leaf, flower and silique. The Fragments Per kb per Million reads (FPKM) values [48] were used to present the $\operatorname{BrCML}$ expression levels. The expression values of genes with abundance of zero were modified to 0.001 for further analysis. The genes with fold change greater than or less than zero were determined as up- or downexpressed genes, respectively. Fold change was calculated as $\log _{2}$ (FPKM in treatment / FPKM in control). Moreover, the microarray and transcriptomic data of Chinese cabbage 'Chiifu' under $45^{\circ} \mathrm{C}$ heat stress [30] and low temperature treatment [31] were obtained from previous reports, respectively. More than two replications were performed in expression analysis. The gene expression levels from different replicates in these papers were calculated according to the previously reported method by Yanai et al. [49]. Clustering analysis of $B r C M L$ genes was performed using Cluster software
[50]. The heat maps of gene expressions were visualized by Java Treeview software [51]. STRING software (http://string-db.org/) [52] was used to generate the interaction networks of Arabidopsis CML genes. The putative interaction networks of $B r C M L$ genes were constructed according to the corresponding homologs between Chinese cabbage and Arabidopsis.

\section{Prediction of microRNAs targeting BrCML genes}

The data of several miRNA libraries were obtained from previous reports on Chinese cabbage [53-56]. The psRNATarget (http://plantgrn.noble.org/psRNATarget/) [57] program was used to predict the putative miRNAs targeting $B r C M L$ genes. The putative regulatory relationships between miRNAs and $B r C M L s$ were analysed and visualized using Cytoscape software [58].

\section{Additional files}

\section{Additional file 1: Table S1. The information on $B r C M L$ genes in Chinese cabbage. (XLSX $22 \mathrm{~kb}$ )}

Additional file 2: Figure S1. The LOGO of four conserved EF-hand motifs among BrCML proteins. (PDF $412 \mathrm{~kb}$ )

Additional file 3: Figure S2. Phylogenetic analysis of CML proteins in Chinese cabbage, Arabidopsis and rice. (PDF 742 kb)

Additional file 4: Table S2. The distribution and syntenic paralogs of BrCML genes in three subgenomes of Chinese cabbage. (XLSX 9 kb)

Additional file 5: Table S3. The orthologous gene pairs ( $E$-value $\leq 1 E-10$ ) in CML proteins of Chinese cabbage, Arabidopsis thaliana and Brassica oleracea. (XLSX $12 \mathrm{~kb}$ )

Additional file 6: Table S4. The FPKM values of BrCML genes in five different tissues of Chinese cabbage. (XLSX 13 kb)

Additional file 7: Table S5. The expression values of $B r C M L$ genes under $45^{\circ} \mathrm{C}$ heat treatment in Chinese cabbage. (XLSX $19 \mathrm{~kb}$ )

Additional file 8: Figure S3. Differential expression patterns of BrCML genes under low temperature stress. 'Heading': the group with a constant $25^{\circ} \mathrm{C}$ temperature; 'non-heading': the group with a $4^{\circ} \mathrm{C}$ low temperature treatment. (PDF $407 \mathrm{~kb}$ )

Additional file 9: Table S6. The expression values of BrCML genes under low temperature treatment in Chinese cabbage. (XLSX 11 kb)

Additional file 10: Table S7. The putative miRNAs targeting BrCML genes from previously reported libraries. (XLSX 9 kb)

Additional file 11: Figure S4. Putative regulatory relationships between candidate miRNAs and BrCML genes. (PDF $76 \mathrm{~kb}$ )

Additional file 12: Table S8. Information of STRING search results for interaction networks of BrCML genes. (XLSX 12 kb)

\section{Abbreviations}

BRAD: Brassica database; CaM: Calmodulin; CML: Calmodulin-like protein; FPKM: Fragments Per kb per Million reads; LF: The least fractionated subgenome; MF1: The medium fractionated subgenome; MF2: The most fractionated subgenome; NJ: Neighbourhood-joining method

Acknowledgements

Not applicable

Funding

This work was supported by grants from the National Key Research and Development Program of China (2016YFD0101701), the Doctoral Scientific Research Fund of Northwest A\&F University (2452016183) and the Fundamental Research Funds for the Central Universities of China 
(2452017075). The funding agencies had no role in the design, analysis, interpretation of the data or writing of the manuscript.

\section{Availability of data and materials}

All data generated or analysed during this study were included in this published article and the additional files.

\section{Authors' contributions}

NS and LZ conceived and designed the study. NS wrote the manuscript. NS, $M Z$ and $L Z$ were responsible for data analysis and revising the manuscript. All authors read and approved the final manuscript.

\section{Ethics approval and consent to participate}

Not applicable. This study has not used plant materials directly.

\section{Consent for publication}

Not applicable

\section{Competing interests}

The authors declare that they have no competing interests.

\section{Publisher's Note}

Springer Nature remains neutral with regard to jurisdictional claims in published maps and institutional affiliations.

\section{Received: 8 June 2017 Accepted: 25 October 2017}

\section{Published online: 02 November 2017}

\section{References}

1. Berridge MJ, Lipp P, Bootman MD, Berridge M, Lipp P, Bootman M. The versatility and universality of calcium signaling. Nat Rev Mol Cell Biol. 2000; $1(1): 11-21$.

2. Hetherington $\mathrm{AM}$, Brownlee $\mathrm{C}$. The generation of $\mathrm{Ca}(2+)$ signals in plants. Annu Rev Plant Biol. 2004;55:401-27.

3. Bergey DR, Kandel R, Tyree BK, Dutt M, Dhekney SA. The role of calmodulin and related proteins in plant cell function: an ever-thickening plot. Springer Sci Rev. 2014:2(1):145-59.

4. Knight H. Calcium signaling during abiotic stress in plants. Int Rev Cytol. 2000:195:269-324.

5. Yang T, Poovaiah BW. Calcium/calmodulin-mediated signal network in plants. Trends Plant Sci. 2003:8(10):505-12.

6. Das R, Pandey GK. Expressional analysis and role of calcium regulated kinases in abiotic stress signaling. Curr Genomics. 2010;11(1):2

7. Defalco TA, Bender KW, Snedden WA. Breaking the code: Ca sensors in plant signaling. Biochem J. 2010;425(1):27-40.

8. Perochon A, Aldon D, Galaud JP, Ranty B. Calmodulin and calmodulin-like proteins in plant calcium signaling. Biochimie. 2011;93(12):2048-53.

9. Gifford JL, Walsh MP, Vogel HJ. Structures and metal-ion-binding properties of the $\mathrm{Ca}^{2+}$-binding helix-loop-helix EF-hand motifs. Biochem J. 2007;405:199-221.

10. Snedden WA, Fromm H. Calmodulin as a versatile calcium signal transducer in plants. New Phytol. 2001;151:35-66.

11. Bouche N, Yellin A, Snedden WA, Fromm H. Plant-specific calmodulinbinding proteins. Annu Rev Plant Biol. 2005;56:435-66.

12. McCormack E, Braam J. Calmodulins and related potential calcium sensors of Arabidopsis. New Phytol. 2003;159:585-98.

13. McCormack E, Tsai Y, Braam J. Handling calcium signaling: Arabidopsis CaMs and CMLs. Trends Plant Sci. 2005;10:383-9.

14. Boonburapong B, Buaboocha T. Genome-wide identification and analyses of the rice calmodulin and related potential calcium sensor proteins. BMC Plant Biol. 2007;7:4

15. Munir S, Khan MR, Song J, Munir S, Zhang Y, Ye Z, et al. Genome-wide identification, characterization and expression analysis of calmodulin-like (CML) proteins in tomato (Solanum lycopersicum). Plant Physiol Biochem 2016:102:167-79.

16. Mohanta TK, Kumar P, Bae HH. Genomics and evolutionary aspect of calcium signaling event in calmodulin and calmodulin-like proteins in plants. BMC Plant Biol. 2017;17:38

17. Delk NA, Johnson KA, Chowdhury NI. Braam J. CML24, regulated in expression by diverse stimuli, encodes a potential $\mathrm{Ca}^{2+}$ sensor that functions in responses to abscisic acid, daylength, and ion stress. Plant Physiol. 2005;139:240-53.
18. Tsai YC, Delk NA, Chowdhury NI, Braam J. Arabidopsis potential calcium sensors regulate nitric oxide levels and the transition to flowering. Plant Signal Behav. 2007;2:446-54.

19. Wang Y, Zhang WZ, Song LF, Zou JJ, Su Z, Wu WH. Transcriptome analyses show changes in gene expression to accompany pollen germination and tube growth in Arabidopsis. Plant Physiol. 2008;148(3):1201.

20. Chen T, Wu X, Chen Y, Li X, Huang M, Zheng M, et al. Combined proteomic and cytological analysis of $\mathrm{Ca}^{2+}$-calmodulin regulation in Picea meyeri pollen tube growth. Plant Physiol. 2009;149:1111-26.

21. Zhou L, Fu Y, Yang Z. A genome-wide functional characterization of Arabidopsis regulatory calcium sensors in pollen tubes. J Integr Plant Biol. 2009;51:751-61.

22. Wang SS, Diao WZ, Yang X, Qiao Z, Wang M, Acharya BR, et al. Arabidopsis thaliana CML25 mediates the $\mathrm{Ca}(2+)$ regulation of $\mathrm{K}(+)$ transmembrane trafficking during pollen germination and tube elongation. Plant Cell Environ. 2015:38(11):2372.

23. Yang $X$, Wang SS, Wang M, Qiao Z; Bao CC, Zhang W. Arabidopsis thaliana, calmodulin-like protein CML24 regulates pollen tube growth by modulating the actin cytoskeleton and controlling the cytosolic Ca2+ concentration. Plant Mol Biol 2014; 86(3): 225-236.

24. Bender KW, Rosenbaum DM, Vanderbeld B, Ubaid M, Snedden WA. The Arabidopsis calmodulin-like protein, CML39, functions during early seedling establishment. Plant J. 2013;76(4):634-47.

25. Magnan F, Ranty B, Charpenteau M, Sotta B, Galaud JP, Aldon D. Mutations in AtCML9, a calmodulin-like protein from Arabidopsis thaliana, alter plant responses to abiotic stress and abscisic acid. Plant J. 2008;56:575-89.

26. Ma W, Smigel A, Tsai YC, Braam J, Berkowitz GA. Innate immunity signaling: cytosolic $\mathrm{Ca}^{2+}$ elevation is linked to downstream nitric oxide generation through the action of calmodulin or a calmodulin-like protein. Plant Physiol. 2008;148:818-28.

27. Wu P, Wang W, Duan W, Li Y, Hou X. Comprehensive analysis of the CDPKSnRK superfamily genes in Chinese cabbage and its evolutionary implications in plants. Front Plant Sci. 2017:8:162

28. Wang $X$, Wang $H$, Wang J, Sun $R$, Wu J, Liu S, et al. The genome of the mesopolyploid crop species Brassica rapa. Nat Genet. 2011;43:1035-9.

29. Tong C, Wang X, Yu J, Wu J, Li W, Huang J, et al. Comprehensive analysis of RNA-seq data reveals the complexity of the transcriptome in Brassica rapa. BMC Genomics. 2013;14:689.

30. Dong X, Yi H, Lee J, Nou I, Han C, Hur Y. Global gene-expression analysis to identify differentially expressed genes critical for the heat stress response in Brassica rapa. PLoS One. 2014;10(6):e0130451.

31. Zhang CW, Wei YP, Xiao D, Gao L, Lyu S, Hou X, et al. Transcriptomic and proteomic analyses provide new insights into the regulation mechanism of low-temperature-induced leafy head formation in Chinese cabbage. J Proteome. 2016:144:1.

32. Rogers K, Chen X. Biogenesis, turnover, and mode of action of plant microRNAs. Plant Cell. 2013:25:2383-99.

33. Lysak MA, Koch MA, Pecinka A, Schubert I. Chromosome triplication found across the tribe Brassiceae. Genome Res. 2005:15:516-25.

34. Cheng F, Mandáková T, Wu J, Xie Q, Lysak M, Wang X. Deciphering the diploid ancestral genome of the Mesohexaploid Brassica rapa. Plant Cell. 2013;25(5):1541-54.

35. Chinpongpanich A, Limruengroj K, Phean-o-pas S, Limpaseni T, Buaboocha $T$. Expression analysis of calmodulin and calmodulin-like genes from rice, Oryza sativa L. BMC Res Notes. 2012;5(1):625.

36. Duval F, Renard M, Jaquinod M, Biou V, Montrichard F, Macherel D. Differential expression and functional analysis of three calmodulin isoforms in germinating pea (Pisum sativum L.) seeds. Plant J. 2002;32:481-93.

37. Zhang K, Yue D, Wei W, Hu Y, Feng J, Zou Z. Characterization and functional analysis of calmodulin and calmodulin-like genes in Fragaria vesca. Front Plant Sci. 2016;7:1820.

38. Vanderbeld B, Snedden WA. Developmental and stimulus-induced expression patterns of Arabidopsis calmodulin-like genes CML37, CML38 and CML39. Plant Mol Biol. 2007:64:683-97.

39. Chiasson D, Ekengren SK, Martin GB, Dobney SL, Snedden WA. Calmodulinlike proteins from Arabidopsis and tomato are involved in host defense against Pseudomonas syringae pv. tomato. Plant Mol Biol. 2005;58:887-97.

40. Vadassery J, Reichelt M, Hause B, Gershenzon J, Boland W, Mithofer A CML42-mediated calcium signaling coordinates responses to Spodoptera herbivory and abiotic stresses in Arabidopsis. Plant Physiol. 2012; 159:1159-75. 
41. Takabatake R, Karita E, Seo S, Mitsuhara I, Kuchitsu K, Ohashi Y. Pathogen induced calmodulin isoforms in basal resistance against bacterial and fungal pathogens in tobacco. Plant Cell Physiol. 2007;48:414-23.

42. Choi HW, Lee DH, Hwang BK. The pepper calmodulin gene CaCaM1 is involved in reactive oxygen species and nitric oxide generation required for cell death and the defense response. Mol Plant-Microbe Interact. 2009;22: 1389-400.

43. Zhang W, Zhou RG, Gao YJ, Zheng SZ, Xu P, Zhang SQ, et al. Molecular and genetic evidence for the key role of AtCaM3 in heat shock signal transduction in Arabidopsis. Plant Physiol. 2009;149:1773-84.

44. Liu HT, Gao F, Li GL, Han JL, Liu DL, Sun DY, et al. The calmodulin binding protein kinase 3 is part of heat-shock signal transduction in Arabidopsis thaliana. Plant J. 2008:55:760-73.

45. Cheng F, Liu S, Wu J, Fang L, Sun S, Liu B, et al. BRAD, the genetics and genomics database for Brassica plants. BMC Plant Biol. 2011:11:136.

46. Kumar S, Stecher G, Tamura K. MEGA7: molecular evolutionary genetics analysis version 7.0 for bigger datasets. Mol Biol Evol. 2016;33(7):1870.

47. Krzywinski M, Schein J, Birol I, Connors J, Gascoyne R, Horsman D, et al. Circos: an information aesthetic for comparative genomics. Genome Res. 2009;19:1639-45.

48. Mortazavi A, Williams BA, McCue K, Schaeffer L, Wold B. Mapping and quantifying mammalian transcriptomes by RNA-Seq. Nat Methods. 2008:5:621-8.

49. Yanai I, Benjamin H, Shmoish M, Chalifa-Caspi V, Shklar M, Ophir R, et al. Genome-wide midrange transcription profiles reveal expression level relationships in human tissue specification. Bioinformatics. 2005;21(5):650-9.

50. de Hoon M, Imoto S, Nolan J, Miyano S. Open source clustering software. Bioinformatics. 2004;20:1453-4.

51. Saldanha AJ. Java Treeview-extensible visualization of microarray data. Bioinformatics. 2004;20:3246-8.

52. Szklarczyk D, Morris JH, Cook H, Kuhn M, Wyder S, Simonovic M, et al. The STRING database in 2017: quality-controlled protein-protein association networks, made broadly accessible. Nucleic Acids Res. 2017;45:D362-8.

53. Kim B, HJ Y, Park SG, Shin JY, Kim MO, Mun JH. Identification and profiling of novel microRNAs in the Brassica rapa genome based on small RNA deep sequencing. BMC Plant Biol. 2012;12(1):218.

54. Wang F, Li L, Liu L, Li H, Zhang Y, Yao Y, et al. High-throughput sequencing discovery of conserved and novel microRNAs in Chinese cabbage (Brassica rapa L. ssp. pekinensis). Mol Gen Genomics. 2012;287(7):555-63.

55. Zhou B, Fan P, Li Y. High-throughput sequence analysis of small RNAs in skotomorphogenic seedlings of Brassica rapa ssp. rapa. Gene. 2014;548(1):68-74.

56. Zhou B, Fan P, Li Y, Yan H, Exploring XQ. miRNAs involved in blue/UV-A light response in Brassica rapa reveals special regulatory mode during seedling development. BMC Plant Biol. 2016;16:111.

57. Dai $X$, Zhao PX. psRNA target: a plant small RNA target analysis server. Nucleic Acids Res. 2011;39:W155-9.

58. Shannon P, Markiel A, Ozier O, Baliga NS, Wang JT, Ramage D, et al. Cytoscape: a software environment for integrated models of biomolecular interaction networks. Genome Res. 2003;13:2498-504.

\section{Submit your next manuscript to BioMed Central and we will help you at every step:}

- We accept pre-submission inquiries

- Our selector tool helps you to find the most relevant journal

- We provide round the clock customer support

- Convenient online submission

- Thorough peer review

- Inclusion in PubMed and all major indexing services

- Maximum visibility for your research

Submit your manuscript at www.biomedcentral.com/submit 